\title{
Neurosurgery and coronavirus: impact and challenges-lessons learnt from the first wave of a global pandemic
}

\author{
Keyoumars Ashkan ${ }^{1,2} \cdot$ Josephine Jung ${ }^{1,2}$ (D) Alexandra Maria Velicu ${ }^{1,2} \cdot$ Ahmed Raslan $^{1} \cdot$ Mohammed Faruque $^{1} \cdot$ \\ Pandurang Kulkarni ${ }^{1}$ - Cristina Bleil $^{1} \cdot$ Harutomo Hasegawa $^{1}$ - Ahilan Kailaya-Vasan ${ }^{1}$ - Eleni Maratos ${ }^{1}$. \\ Gordan Grahovac ${ }^{1}$. Francesco Vergani ${ }^{1}$ - Bassel Zebian ${ }^{1}$. Sinan Barazi ${ }^{1} \cdot$ Irfan Malik $^{1}$ • David Bell ${ }^{1}$. Daniel Walsh ${ }^{1}$.

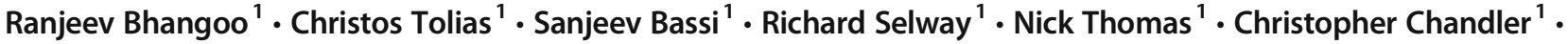 \\ Richard Gullan ${ }^{1}$
}

Received: 22 September 2020 / Accepted: 12 November 2020 / Published online: 21 November 2020

(C) The Author(s) 2020

\begin{abstract}
Introduction and objectives The novel severe acute respiratory syndrome coronavirus 2 (COVID-19) pandemic has had drastic effects on global healthcare with the UK amongst the countries most severely impacted. The aim of this study was to examine how COVID-19 challenged the neurosurgical delivery of care in a busy tertiary unit serving a socio-economically diverse population. Methods A prospective single-centre cohort study including all patients referred to the acute neurosurgical service or the subspecialty multidisciplinary teams (MDT) as well as all emergency and elective admissions during COVID-19 (18th March 2020-15th May 2020) compared to pre-COVID-19 (18th of January 2020-17th March 2020). Data on demographics, diagnosis, operation, and treatment recommendation/outcome were collected and analysed.

Results Overall, there was a reduction in neurosurgical emergency referrals by $33.6 \%$ and operations by $55.6 \%$ during the course of COVID-19. There was a significant increase in the proportion of emergency operations performed during COVID-19 (75.2\% of total, $n=155)$ when compared to pre-COVID-19 $(n=198,43.7 \%$ of total, $p<0.00001)$. In contrast to other published series, the 30-day perioperative mortality remained low $(2.0 \%)$ with the majority of post-operative COVID-19-infected patients $(n=13)$ having underlying medical co-morbidities and/or suffering from post-operative complications.

Conclusion The capacity to safely treat patients requiring urgent or emergency neurosurgical care was maintained at all times. Strategies adopted to enable this included proactively approaching the referrers to maintain lines of communications, incorporating modern technology to run clinics and MDTs, restructuring patient pathways/facilities, and initiating the delivery of NHS care within private sector hospitals. Through this multi-modal approach we were able to minimize service disruptions, the complications, and mortality.
\end{abstract}

Keywords Coronavirus $\cdot$ Emergency referrals $\cdot$ Neurosurgery $\cdot$ Pandemic

\section{Introduction}

King's College Hospital NHS Foundation Trust (KCH), built in 1840 , is one of the largest teaching hospitals in the UK,

This article is part of the Topical Collection on Infection

Josephine Jung

Josephine.Jung@nhs.net

1 Department of Neurosurgery, King's College Hospital, Denmark Hill, London SE5 9RS, UK

2 Neurosciences Clinical Trials Unit, King's College Hospital, London, UK serving a local inner-city population of 700,000 in the London districts of Southwark and Lambeth. The tertiary neurosurgical service is amongst the busiest in the country covering a regional catchment population of approximately 4 million across South East London and the county of Kent [13].

The local London boroughs have a multi-ethnic population with a comparatively high proportion of Black people (25.9\%), compared to the whole London (10.9\%). Amongst the largest ethnic minority groups are Black African (16.1\%) and Black Caribbean (8.0\%). Approximately half of the local population identifies as White British (52.2\%), much lower than the national average. The socio-economic profile of the local population shows the lowest level of employment amongst all London districts [16, 21]. 
The novel severe acute respiratory syndrome coronavirus 2 (COVID-19) pandemic had a drastic effect on global healthcare and Europe was at the epicentre of the pandemic from March to May 2020. The UK had one of the highest death tolls across Europe and London was the worst affected. $\mathrm{KCH}$ had at its peak 517 inpatients with COVID-19 of which 96 were being cared for in the intensive treatment unit (ITU).

The GlobalSurg group recently published that surgical services, both elective and emergency, were severely impacted by COVID-19 and that there was an increased 30-day mortality of up to $23.8 \%$ in patients infected with COVID-19 [2]. The aim of the paper here was to examine specifically the impact of the COVID-19 pandemic on the neurosurgical delivery of care in a busy unit serving a socio-economically challenging population. We also examined the patient outcomes as well as described the strategies adopted to allow safe delivery of neurosurgical services.

\section{Methods}

\section{Study design}

On 18th March 2020, our unit entered the acute "COVID-19" phase, demarcated by the day of the first pre-operatively suspected COVID-19 infection in a neurosurgical patient, and when neurosurgical rota and service changes were adopted. The phase officially ended on 15th May (59 days later) when our hospital, in line with the NHS directive, entered the recovery phase. During the COVID-19 period, data were collected prospectively on all patients referred to the acute neurosurgical service, patients who were admitted electively, and patients referred to subspecialty multidisciplinary teams (MDT). These data were then compared to those obtained in the immediately preceding 59-day period (18th January to 17th March), the "pre-COVID-19", to assess the impact of the pandemic. The manuscript was written following the Strengthening the Reporting of Observational Studies in Epidemiology (STROBE) checklist [24].

\section{Data collection and outcome measures}

Data on the acute neurosurgical referrals were obtained through an online Patient Care System (PCS) and data on referrals to the MDTs were obtained from the relevant coordinators. Emergency and elective operating lists were sourced through the software "Galaxy Operating Theatres". Electronic patient records were accessed to capture the following: age, gender, diagnosis, type of procedure (emergency vs. elective, adult vs. paediatric, cranial vs. spinal), subspecialty (functional, neuro-oncology, trauma, neurovascular, skull base, spinal, paediatric neurosurgery), COVID-19 infection (pre-operatively vs. post-operatively), and post-operative complications. For
COVID-19-infected patients, data on ethnicity, co-morbidities, perioperative complications, ITU/hospital stay, discharge destination, and mortality were also included. Additionally, referral data included type of referral (new vs. follow-up), pathology, MDT outcome, and treatment delay/ recommendation.

\section{Rota change, guidelines, and operating theatre adjustments}

Rota and service provision changes were put in place during the pandemic on 18th of March 2020. Given the rapidly evolving Public Health England [20], NHS England [15], and the Society of British Neurological Surgeons guidelines [22], a local KCH Neurosurgery working group was established to actively review the evidence and synthesize a Guidance [12] for adjustments to elective and emergency operations, theatre ventilation, and use of personal protective equipment (PPE).

$\mathrm{KCH}$ had its first two COVID-19-positive patients on 04th of March 2020 (Fig. 1-day 0) and reached its peak on the 8th of April 2020 with 517 COVID-19-positive inpatients of which 96 were in ITU. The first change to our neurosurgical practice was put in place on 18th March 2020 where the rota was revised from a subspecialty team-based system (consisting of 1-2 neurosurgical trainees working for a specific consultant) to a twilight rota with 1 "green" team (no COVID-19 patient contact), 1 "red" team (COVID-19-positive/suspected patient contact), and 1 stand-by team in case other team members fell ill and had to consequently self-isolate. Consultant and trainee rotas both switched to a 24-h shift with one on-site consultant at all times and another on-call in reserve.

The three daily elective operating lists were condensed into one list per day from the 23rd of March 2020. Throughout the pandemic, one emergency neurosurgical theatre remained active. The allocation of theatre resources was undertaken by the Executive board of the hospital. The neurosurgical lists were arranged/triaged by the on-call neurosurgery consultant on the day. Trust guidelines recommended that ventilation in both laminar flow and conventionally ventilated theatres should remain fully switched on during surgical procedures where patients may have COVID-19 infection. All operations were performed with full PPE, including either FFP3 mask (for aerosol-generating procedures) or fluid-resistant mask (nonaerosol-generating procedures), hat, visor, gloves and fluidresistant disposable gown. Staff training for proper donning and doffing was mandatory.

Our inpatient neurosurgical service usually consists of one purely elective 31-bedded ward, three wards for emergency and additional elective neurosurgical patients ( $~ 50-60$ beds), and one dedicated 12-bedded neurosurgery high dependency unit (HDU). During COVID-19, out of these, one emergency 
Fig. 1 This graph describes the number of patients admitted to our hospital with COVID-19 infection. Overall number of inpatients is depicted in black, and the number of patients in intensive care is provided in grey. Day 0 was the 04th of March 2020
Covid-19 positive inpatients

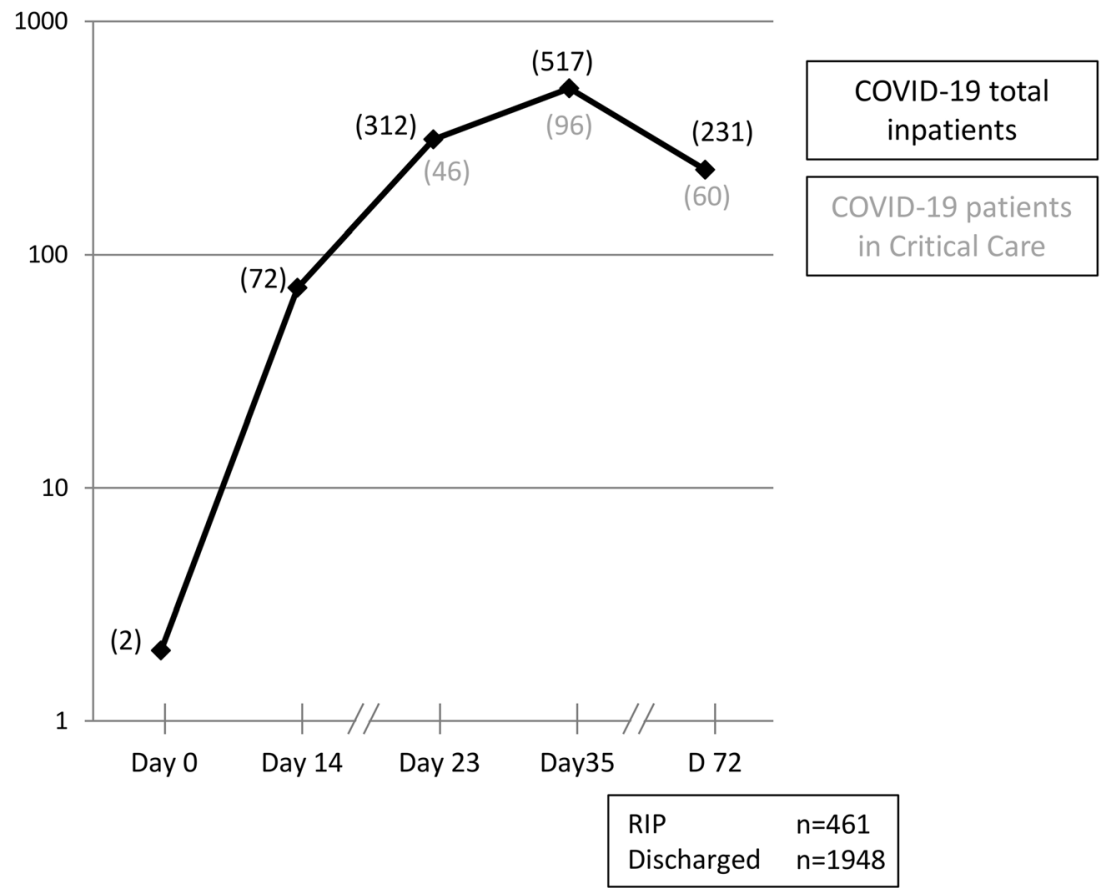

admission ward and its adjacent HDU were closed to neurosurgery admissions to provide capacity for the hospital's general COVID-19 patients. The HDU was relocated to replace one of the other neurosurgical wards with level 2 bed numbers expanded to 32. The remaining two neurosurgical wards were divided into "dirty" (COVID-19 positive) and "clean" (nonCOVID-19) wards (Fig. 2).

Nearly all face-to-face outpatient appointments were cancelled and essential appointments were conducted via phone consultations. The MDT referral process remained unchanged; however, meetings took place virtually via Microsoft $\circledast$ Teams (Redmond, USA) from 01st April 2020.

\section{Statistical analysis}

Descriptive statistics were used to characterize the patient population. Statistical analysis was performed using GraphPad Prism V7. Chi-squared test and the Mann-

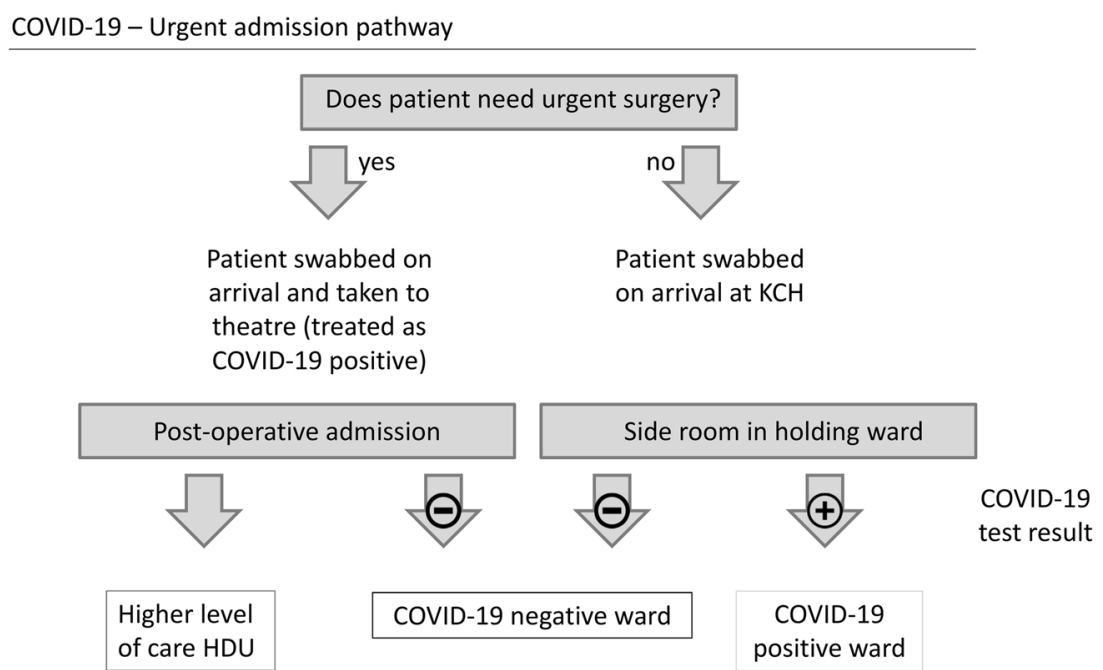

Fig. 2 Flowchart describing admission pathway for patients requiring urgent treatment during COVID-19. All neurosurgical patients were swabbed for COVID-19 upon arrival at $\mathrm{KCH}$. If urgent surgery was required, they were taken to theatre and treated as COVID-19 positive until the test result was available. If urgent surgery was not required, they were isolated in a side room in a dedicated holding ward until the COVID-19 test result was available and then either cared for in a COVID-19-positive ward or allocated to a COVID19-negative ward based on the results 
Whitney $U$ test were used to assess the statistical significance of observed differences between cohorts before and during the COVID-19 pandemic.

\section{Results}

\section{Emergency referrals during COVID-19}

Pre-COVID-19, the median number of new acute referrals was 31 (range 17-45) per $24 \mathrm{~h}$. During COVID-19, this decreased to 21 (range 10-34) per day. There was a statistically significant reduction in the overall number of referrals from 1847 to 1227 (Table $1 ; p<0.05$ ).

Subspecialty emergency referrals changed to proportionately fewer skull base and spinal referrals, but proportionately increased trauma, vascular, oncology, and paediatric referrals (Table $1 ; p<0.01$ ). Approximately $10 \%$ of patients referred as an emergency pre-COVID-19 $(n=190)$ and during COVID$19(n=119)$ were accepted for emergency transfer. There was no change to the definition of what constituted an emergency during or pre-COVID-19, namely being a condition that was life or limb threatening within a matter of days if left untreated. There was no significant change in the proportion of patients with neurological deficit, GCS $\leq 8$, or age $>65$ years being transferred to our neurosurgical centre $(p>0.05)$.

In fact, there was no statistically significant difference in age amongst patients that were admitted pre-COVID-19 (median age 53 (range 0-92) years) and during COVID-19 (median age 51 (range 0-89) years. There was however a change in gender of patients admitted pre-COVID-19 and during COVID-19 with proportionately more males being admitted during COVID-19 (59.67\% compared to $52.31 \%$ preCOVID-19, $p<0.05$ ). This may potentially reflect the generally more health averse and risk-prone occupational and nonoccupational behaviour amongst men resulting in acute admissions. The distribution of ethnic minority patients admitted pre-COVID-19 (8.15\% Black, 3.94\% Asian, 0.41\% Hispanic) and during COVID-19 (9.54\% Black, 3.54\% Asian, 0.54\% Hispanic) remained stable, albeit in a higher proportion of patients the ethnicity was not recorded during COVID-19 (24.73\% pre-COVID-19, 36.24\% during COVID-19), possibly reflecting the limited availability of hospital administrative support staff to record these during COVID-19.

\section{Emergency and elective neurosurgical operations performed before and during COVID-19}

The total number of operations decreased from $n=453$ (preCOVID-19) to $n=206$ (Table $1 ; p<0.0001$ ) with the daily median number of operations decreasing from 8 to 3 . A higher percentage of emergency operations was performed during COVID-19 (75.2\% of total, $n=155)$ compared to pre-
COVID-19 $(n=198,43.7 \%$ of total, $p<0.00001)$. There was no significant change in the proportion of cranial versus spinal operations (Table 1). Overall, significantly fewer patients aged $>65$ years underwent an operation during COVID-19 $(p<0.01)$. The operations for adults and paediatrics per neurosurgical subspecialty changed significantly $(p<$ 0.01 ) with subspecialties with a high proportion of elective work, such as functional, skull base, and spinal neurosurgery, being affected the most.

Tables 2 and 3 summarize the data on patients undergoing neurosurgery in adult and paediatric cases, respectively. The total number of adult operations performed dropped from $n=$ 408 to $n=173$ during COVID-19, with a significant amount of functional and degenerative spinal neurosurgical work being deferred or cancelled ( $p<0.01$; Table 2$)$. The number of operations amongst the emergency subspecialties, such as trauma and vascular neurosurgery, also decreased during COVID-19 by approximately 50\%; however, the case mix remained similar. The most common traumatic pathologies requiring emergency operation were chronic subdural hematoma (pre-COVID-19 $n=26$, COVID-19 $n=19$ ), vertebral fracture (pre-COVID-19 $n=11$, COVID-19 $n=5$ ), and acute subdural hematoma (pre-COVID-19 $n=9$, COVID-19 $n=3$ ). Although all the numbers decreased, the smallest drop in cases was amongst surgeries for chronic subdural haematomas, possibly related to their relatively more chronic presentation. Similarly, vascular operations decreased with fewer aneurysms being clipped during COVID-19 $(n=1)$ compared to pre-COVID-19 $(n=9)$.

Within the neuro-oncology service, the overall number of operations decreased from $n=60$ (14.7\% of total) to $n=31$ (17.9\% of total) during COVID-19. Similarly, the number of craniotomies for high-grade gliomas decreased from $n=31$ (7.6\% of total) to $n=12$ (6.9\% of total) during the pandemic.

Our skull base service was severely affected during COVID-19 with only 3 operations for pituitary adenoma/ apoplexy being performed during COVID-19 (1.7\% of total) from a previous number of 16 operations pre-COVID-19 (3.9\% of total). No operations for trigeminal neuralgia, vestibular schwannoma, or chiari malformation were performed during COVID-19.

In functional neurosurgery, no new implantations for deep brain stimulation (DBS), spinal cord stimulation, or occipital nerve stimulation were performed. The battery change service for patients with movement disorders, however, continued albeit in the day case setting (DBS battery change $n=5$ in both periods). All spinal surgeries decreased during COVID19; however, notably, operations for cauda equina syndrome (pre-COVID-19 $n=26$, COVID-19 $n=14$ ) and myelopathies (pre-COVID-19 $n=28$, COVID-19 $n=8$ ) were reduced by $\geq$ $50 \%$ during COVID-19, whereas operations within spinal oncology category remained stable (pre-COVID-19 $n=14$, COVID-19 $n=15$ ). 
Table 1 Characteristics of emergency referrals and operations

\begin{tabular}{|c|c|c|c|}
\hline Period & Pre-COVID-19 & COVID-19 & $p$ value \\
\hline Emergency referrals, total (\%) & $1847(100.0)$ & $1227(100.0)$ & $p<0.01$ \\
\hline Trauma/vascular & $956(51.8)$ & $656(53.5)$ & \\
\hline Oncology & $210(11.4)$ & $171(13.9)$ & \\
\hline Skull base & $36(1.9)$ & $12(1.0)$ & \\
\hline Spinal & $428(23.2)$ & $229(18.7)$ & \\
\hline Paediatrics & $75(4.1)$ & $59(4.8)$ & \\
\hline Other* & $141(7.6)$ & $100(8.1)$ & \\
\hline Emergency transfer, total (\%) & $190(100.0)$ & $119(100.0)$ & $p>0.05$ \\
\hline Neurological deficit & $92(48.4)$ & $55(46.2)$ & \\
\hline $\mathrm{GCS}^{\mathrm{a}} \leq 8$ & $12(6.3)$ & $10(8.4)$ & \\
\hline Age $>65$ years & $42(22.1)$ & $21(17.6)$ & \\
\hline Surgery, total $(\%)$ & $453(100.0)$ & $206(100.0)$ & \\
\hline $\begin{array}{l}\text { Emergency } \\
\text { Elective }\end{array}$ & $\begin{array}{l}198(43.7) \\
255(56.3)\end{array}$ & $\begin{array}{l}155(75.2) \\
51(24.8)\end{array}$ & $p<0.00001$ \\
\hline $\begin{array}{l}\text { Cranial } \\
\text { Spinal }\end{array}$ & $\begin{array}{l}263(58.1) \\
190(41.9)\end{array}$ & $\begin{array}{l}134(65.0) \\
72(35.0)\end{array}$ & ns \\
\hline $\begin{array}{l}\text { Adult } \\
\text { Paediatric }\end{array}$ & $\begin{array}{l}408(90.1) \\
45(9.9)\end{array}$ & $\begin{array}{l}173(84.0) \\
33(16.0)\end{array}$ & $p<0.05$ \\
\hline Age at operation (years) & & & $p>0.05$ \\
\hline Mean \pm SD & $50 \pm 21$ & $47 \pm 22$ & \\
\hline Range & $0-92$ & $0-86$ & \\
\hline Operative age groups (years) & & & $p<0.01$ \\
\hline $0-17$ & 44 & 33 & \\
\hline $18-65$ & 292 & 178 & \\
\hline$>65$ & 117 & 41 & \\
\hline \multicolumn{4}{|l|}{ COVID-19 infection } \\
\hline Pre-operatively & 0 & 4 & \\
\hline Post-operatively (during inpatient stay) & 7 & 6 & \\
\hline Mortality, total (\% of operations) & $5(1.1)$ & $4(2.0)$ & \\
\hline 30-day perioperative (emergencies) & $5(2.5)$ & $3(1.9)$ & \\
\hline 30-day perioperative (electives) & $0(0.0)$ & $1(2.2)$ & \\
\hline \multicolumn{3}{|c|}{ Operations per subspecialty (adult and paediatric) } & $p<0.01$ \\
\hline Trauma/vascular & $75(16.6)$ & $44(21.4)$ & \\
\hline Oncology & $67(14.8)$ & $40(19.4)$ & \\
\hline Skull base & $37(8.2)$ & $8(3.9)$ & \\
\hline Spinal & $149(32.9)$ & $55(26.7)$ & \\
\hline Functional & $43(9.5)$ & $8(3.9)$ & \\
\hline Other* & $82(18.1)$ & $51(24.8)$ & \\
\hline
\end{tabular}

*Emergency referrals and operations for other reasons such as hydrocephalus and infection were excluded from statistic calculation

${ }^{\text {a }}$ Glasgow coma scale

\section{Neurovascular referral service}

The total number of referrals to the vascular MDT decreased from $n=245$ to $n=161$ during COVID-19 $(p<0.05)$. The total number of patients referred with an intracranial aneurysm decreased from $n=185$ (75.5\% of total pre-COVID-19) to $n=$ 132 (82.0\% of total during COVID-19; Table 4). Within that group, referred unruptured symptomatic aneurysms remained approximately stable ( 2.5 of total). The number of AVMs referred decreased from $n=34$ (13.9\% of total pre-COVID19) to $n=8(5.0 \%$ of total during COVID-19).

Sixteen patients underwent emergency treatment preCOVID-19, 6 of those underwent open surgery and 10 underwent endovascular treatment. During COVID-19, only 
Table 2 Number and composition of adult operations performed by subspecialty

\begin{tabular}{|c|c|c|c|}
\hline Adult & Pre-COVID-19 & COVID-19 & $p$ value \\
\hline Number of operations in $N$ ( $\%$ of total) & $408(100.0)$ & $173(100.0)$ & \\
\hline Functional & $37(9.1)$ & $8(4.6)$ & $p<0.01$ \\
\hline $\mathrm{DBS}^{\mathrm{a}}$ for Parkinson's disease/tremor & $8(2.0)$ & $0(0.0)$ & \\
\hline Intractable epilepsy/VNS ${ }^{\mathrm{b}}$ & $18(4.4)$ & $1(0.6)$ & \\
\hline Peripheral nerve & $3(0.7)$ & $1(0.6)$ & \\
\hline Baclofen pump/other & $2(0.4)$ & $1(0.6)$ & \\
\hline DBS battery change & $5(2.7)$ & $5(2.9)$ & \\
\hline Spinal & $145(35.5)$ & $51(29.5)$ & $p<0.01$ \\
\hline Myelopathy & $28(6.9)$ & $8(4.6)$ & \\
\hline Radiculopathy & $73(17.9)$ & $13(7.5)$ & \\
\hline Cauda equina syndrome & $26(6.4)$ & $14(8.1)$ & \\
\hline $\mathrm{MSCC}^{\mathrm{d}}$ and spinal tumour & $14(3.4)$ & $15(8.7)$ & \\
\hline Spinal haematoma and other & $4(1.0)$ & $1(0.6)$ & \\
\hline Trauma & $50(12.3)$ & $31(17.9)$ & $p>0.05$ \\
\hline Acute subdural hematoma & $9(2.2)$ & $3(1.7)$ & \\
\hline Chronic subdural hematoma & $26(6.4)$ & $19(11.0)$ & \\
\hline Extradural hematoma & $4(1.0)$ & $2(1.2)$ & \\
\hline Traumatic brain injury/other & $0(0.0)$ & $1(0.6)$ & \\
\hline Traumatic vertebral fracture & $11(2.7)$ & $5(2.9)$ & \\
\hline Vascular & $22(5.4)$ & $9(5.2)$ & $p>0.05$ \\
\hline Aneurysm & $9(2.2)$ & $1(0.6)$ & \\
\hline Intracranial haemorrhage & $5(1.2)$ & $5(2.9)$ & \\
\hline Ischemic stroke & $1(0.2)$ & $1(0.6)$ & \\
\hline Arteriovenous malformation & $7(1.7)$ & $0(0.0)$ & \\
\hline Arteriovenous fistula & $1(0.2)$ & $2(1.2)$ & \\
\hline Oncology & $60(14.7)$ & $31(17.9)$ & $p>0.05$ \\
\hline Low-grade glioma & $4(1.0)$ & $1(0.6)$ & \\
\hline High-grade glioma & $31(7.6)$ & $12(6.9)$ & \\
\hline Cerebral metastasis & $6(1.5)$ & $6(3.5)$ & \\
\hline Meningioma & $13(3.2)$ & $7(4.0)$ & \\
\hline Other & $6(1.5)$ & $5(2.9)$ & \\
\hline Skull base & $36(8.8)$ & $7(4.0)$ & $p>0.05$ \\
\hline Pituitary adenoma/apoplexy & $16(3.9)$ & $3(1.7)$ & \\
\hline Sphenoid wing meningioma & $5(1.2)$ & $2(1.2)$ & \\
\hline Vestibular schwannoma & $6(1.2)$ & $0(0.0)$ & \\
\hline Chiari malformation & $5(1.2)$ & $0(0.0)$ & \\
\hline Chondrosarcoma & $2(0.5)$ & $0(0.0)$ & \\
\hline Trigeminal neuralgia & $1(0.2)$ & $0(0.0)$ & \\
\hline Craniopharyngioma & $1(0.2)$ & $2(1.2)$ & \\
\hline Other & $58(14.2)$ & $37(21.4)$ & $p>0.05$ \\
\hline Hydrocephalus & $30(7.4)$ & $18(10.4)$ & \\
\hline Primary infections & $7(1.7)$ & $4(2.3)$ & \\
\hline Secondary infections & $15(3.7)$ & $11(6.4)$ & \\
\hline Post-operative hematoma & $2(0.5)$ & $0(0.0)$ & \\
\hline CSF leak/pseudomeningocele & $4(1.0)$ & $4(2.3)$ & \\
\hline
\end{tabular}

${ }^{\text {a }}$ Deep brain stimulation

${ }^{\mathrm{b}}$ Vagal nerve stimulator

${ }^{\mathrm{c}}$ Metastatic spinal cord compression

${ }^{\mathrm{d}}$ Cerebrospinal fluid 
Table 3 Number and composition of paediatric operations performed by subspecialty

\begin{tabular}{llll}
\hline Paediatric & Pre-COVID-19 & COVID-19 & $p$ value \\
\hline Number of operations in $N$ (\% of total) & $45(100.0)$ & $33(100.0)$ & \\
Functional (intractable epilepsy) & $6(13.3)$ & $0(0.0)$ & \\
Spinal & $4(8.9)$ & $4(12.1)$ & $p>0.05$ \\
Myelomeningocele & $3(6.7)$ & $4(12.1)$ & \\
Tethered cord & $1(2.2)$ & $0(0.0)$ & \\
Trauma & $2(4.4)$ & $5(15.2)$ & $p>0.05$ \\
Acute subdural hematoma & $0(0.0)$ & $1(3.0)$ & \\
Extradural hematoma & $1(2.2)$ & $0(0.0)$ & \\
Traumatic brain injury/intracranial haemorrhage & $0(0.0)$ & $3(9.1)$ & \\
Traumatic vertebral fracture & $1(1.1)$ & $1(3.0)$ & \\
Vascular (cavernoma) & $1(2.2)$ & $9(0.0)$ & \\
Oncology & $7(15.6)$ & $4(27.3)$ & \\
LGG & $1(2.2)$ & $2(6.1)$ & \\
HGG & $5(11.1)$ & $2(6.1)$ & \\
Medulloblastoma & $1(2.2)$ & $1(3.0)$ & \\
Ependymoma & $0(0.0)$ & $1(3.0)$ & \\
Skull base (chiari malformation) & $1(2.2)$ & $14(42.4)$ & \\
Other & $24(53.3)$ & $14(42.4)$ & \\
Hydrocephalus & $20(44.4)$ & $2(4.4)$ & $0(0.0)$ \\
Primary infections & $2(4.4)$ & \\
Secondary infections & & \\
\hline
\end{tabular}

1 patient underwent emergency open surgery and 17 patients underwent emergency endovascular treatment. All elective surgery was halted during COVID-19 (Table 4).

\section{Neuro-oncology and skull base referral service}

The total number of referrals to the neuro-oncology MDT decreased from $n=443$ to $n=275$ during COVID-19 ( $p<$ $0.05)$ with the median number of referrals per MDT dropping from $53 \pm 11.63$ to $37 \pm 9.58$. There was no significant change in the ratio of new to follow-up referrals during these periods. Equally, there was no significant change in the treatment recommendation provided for patients with high-grade gliomas (HGG), low-grade gliomas (LGG), and cerebral metastases (CM) $(p>0.05$; Table 5). However, there was a significant treatment delay (surgery or adjuvant therapy), $n=4$ patients ( $0.9 \%$ of total) pre-COVID-19 versus $n=32$ patients $(11.6 \%$ of total, $p<0.00001)$ during COVID-19, with patients with a meningioma affected more severely $(n=16$ overall) compared to patients with gliomas or malignant tumours ( $n=7 \mathrm{HGG}, n$ $=2 \mathrm{LGG}, n=3 \mathrm{CM}$ ). The most common reasons for treatment delay were surgery delay due to COVID-19 because of resource limitations $(n=26)$, secondly unrelated reasons $(n=$ $7)$, patient preference due to fear of infection $(n=2)$ and chemotherapy delay due to COVID-19 $(n=2)$.

Within the skull base service, the number of referrals was significantly reduced from $n=329$ to $n=101$ during COVID-
19 ( $p<0.001)$. Notably, the overall number of patients referred for a pituitary adenoma reduced from $n=80$ to $n=31$ ( $p<$ $0.001)$. Out of those, $n=11$ were referred with pituitary apoplexy pre-COVID-19 and $n=3$ during COVID-19. There was no statistically significant difference in treatment recommendation between patients referred pre-COVID-19 and during COVID-19 for patients with vestibular schwannoma and pituitary adenoma/apoplexy. However, in a higher proportion of patients referred with a meningioma during COVID-19, active treatments such as surgery or SRS, instead of monitoring were recommended, possibly indicating that larger or more clinically symptomatic lesions were being referred during the COVID-19 period ( $p<0.05$; Table 5). All meningioma cases, where specialist intervention was recommended, were located in the medial sphenoid wing. Surgery was recommended to 3 patients with pituitary adenomas during COVID-19: 1 had pituitary apoplexy, 1 had progressively deteriorating vision, and in 1 patient, the pituitary mass had progressed over a short period of time and turned out to be a metastasis. Surgical intervention was deferred in $n=5$ for sphenoid wing meningiomas, and $n=$ 15 for pituitary adenoma.

\section{Referrals to the spinal MDT}

The total number of referrals to the spinal MDT decreased significantly during COVID-19 from $n=526$ to $n=248$ ( $p$ $<0.001$; Table 4 ). The proportion of patients referred with 
Table 4 Referrals to neurovascular and spinal multidisciplinary teams

\begin{tabular}{|c|c|c|c|}
\hline Period & Pre-COVID-19 & COVID-19 & $p$ value \\
\hline Vascular referral age groups (years) & & & $p<0.01$ \\
\hline $0-17$ & 7 & 5 & \\
\hline $18-65$ & 183 & 97 & \\
\hline$>65$ & 55 & 59 & \\
\hline Vascular diagnosis, total (\%) & $245(100.0)$ & $161(100.0)$ & $p<0.01$ \\
\hline Aneurysm(s) & $185(75.5)$ & $132(82.0)$ & \\
\hline Previously ruptured & $79(32.2)$ & $22(13.7)$ & \\
\hline Unruptured symptomatic & $6(2.4)$ & $4(2.5)$ & \\
\hline Unruptured incidental & $100(40.8)$ & $106(65.8)$ & \\
\hline $\mathrm{AVM}^{\mathrm{a}}$ & $34(13.9)$ & $8(5.0)$ & \\
\hline Previously ruptured cranial & $12(4.9)$ & $1(0.6)$ & \\
\hline Unruptured cranial & $20(44.4)$ & $7(4.3)$ & \\
\hline Spinal & $2(0.8)$ & $0(0.0)$ & \\
\hline Cavernoma & $1(0.4)$ & $5(3.1)$ & \\
\hline Other* & $25(10.2)$ & $17(10.6)$ & \\
\hline \multicolumn{4}{|l|}{ Vascular treatment } \\
\hline Emergency, clip/coil & $6 / 10$ & $1 / 17$ & $p>0.05$ \\
\hline Ruptured or dissecting intracranial aneurysm & $4 / 6$ & $1 / 13$ & \\
\hline Ruptured or symptomatic $\mathrm{AVM}^{\mathrm{a}}$ or $\mathrm{AVF}^{\mathrm{b}}$ & $2 / 4$ & $0 / 4$ & \\
\hline Elective, clip/coil & $8 / 9$ & $0 / 0$ & \\
\hline Intracranial aneurysms & $5 / 8$ & $0 / 0$ & \\
\hline $\mathrm{AVM}^{\mathrm{a}}$ or $\mathrm{AVF}^{\mathrm{b}}$ & $3 / 1$ & $0 / 0$ & \\
\hline Spinal MDT ${ }^{\mathrm{c}}$ referrals, total $(\%)$ & $526(100.0)$ & $248(100.0)$ & $p<0.001$ \\
\hline Cauda equina syndrome & $21(4.0)$ & $26(10.5)$ & \\
\hline Degenerative spine & $505(96.0)$ & $222(89.5)$ & \\
\hline Spinal treatment recommendation ( $\%$ of total) & & & $p>0.05$ \\
\hline Routine outpatient & $334(63.5)$ & $151(60.9)$ & \\
\hline Urgent outpatient & $24(4.6)$ & $7(2.8)$ & \\
\hline Conservative or other & $168(31.9)$ & $90(36.3)$ & \\
\hline
\end{tabular}

*Intracranial haemorrhage — no abnormality, non-aneurysmal subarachnoid haemorrhage, stenosis, family history

${ }^{\text {a }}$ Arteriovenous malformation

${ }^{\mathrm{b}}$ Arteriovenous fistula

${ }^{\mathrm{c}}$ Multidisciplinary team

cauda equina syndrome increased significantly from $4.0 \%(n$ $=21)$ to $10.5 \%(n=26, p<0.001)$. There was no statistically significant difference between spinal MDT treatment recommendations before and during COVID-19 (Table 4).

\section{Functional and paediatric neurosurgery}

The number of functional neurosurgery MDTs was reduced from twice-weekly pre-COVID-19 to 3 during COVID-19. No elective functional neurosurgery took place during COVID-19 although battery replacement for movement disorder patients continued (Table 2).

In the paediatric service, the total number of operations performed was not as severely affected as the adult service
(pre-COVID-19 $n=45$, during COVID-19 $n=33$ ) but the case load amongst the subspecialties changed (Table 3 ). In particular, trauma cases increased from $n=2(4.4 \%$ of total pre-COVID-19) to $n=5$ (15.2\% of total during COVID-19). Oncology operations also increased from $n=7$ (15.6\% of total) to $n=9$ (27.3\% of total) during COVID-19. No functional or neurovascular operations were performed during COVID-19 within our paediatric cohort.

\section{Surgical outcomes and COVID-19 infections in neuro- surgical patients}

Overall, 30-day perioperative mortality remained low during COVID-19 $(n=4,2.0 \%)$ compared to pre-COVID-19 $(n=5$, 
Table 5 Referrals to neurooncology and skull base multidisciplinary teams

\begin{tabular}{|c|c|c|c|}
\hline Period & Pre-COVID-19 & COVID-19 & $p$ value \\
\hline Neuro-oncology diagnosis, total (\%) & $443(100.0)$ & $276(100.0)$ & $p>0.05$ \\
\hline New referrals & $298(67.3)$ & $185(67.0)$ & \\
\hline High-grade glioma & $65(14.7)$ & $33(12.0)$ & \\
\hline Low-grade glioma & $22(5.0)$ & $9(3.3)$ & \\
\hline Cerebral metastasis & $80(18.1)$ & $60(21.7)$ & \\
\hline Meningioma & $59(13.3)$ & $37(13.4)$ & \\
\hline Other* & $72(16.3)$ & $46(16.7)$ & \\
\hline Follow-up (including post-operative) & $145(32.7)$ & $91(33.0)$ & \\
\hline High-grade glioma & $34(7.7)$ & $26(9.4)$ & \\
\hline Low-grade glioma & $11(2.5)$ & $8(2.9)$ & \\
\hline Cerebral metastasis & $41(9.3)$ & $32(11.6)$ & \\
\hline Meningioma & $35(7.9)$ & $17(6.2)$ & \\
\hline Other* & $24(5.4)$ & $8(2.9)$ & \\
\hline \multicolumn{4}{|l|}{ Treatment recommendation } \\
\hline High-grade glioma, total & 99 & 59 & $p>0.05$ \\
\hline Surgery, $\%$ & $32(32.3)$ & $12(20.3)$ & \\
\hline Monitoring, conservative or other, $\%$ & $67(67.7)$ & $47(79.7)$ & \\
\hline Low-grade glioma, total & 33 & 17 & $p>0.05$ \\
\hline Surgery, $\%$ & $6(18.2)$ & $4(23.5)$ & \\
\hline Monitoring, conservative or other, $\%$ & $27(81.8)$ & $13(76.5)$ & \\
\hline Cerebral metastasis, total & 121 & 92 & $p>0.05$ \\
\hline Intervention (surgery/SRS ${ }^{\mathrm{a}}$ ), \% & $30(7 / 23)(24.8)$ & $32(6 / 26)(34.8)$ & \\
\hline Monitoring, conservative or other, $\%$ & $91(75.2)$ & $60(65.2)$ & \\
\hline Skull base diagnosis, total (\%) & $329(100.0)$ & $101(100.0)$ & $p<0.05$ \\
\hline New referrals & $112(34.0)$ & $48(47.5)$ & \\
\hline Meningioma & $25(7.6)$ & $14(13.9)$ & \\
\hline Vestibular schwannoma & $17(5.2)$ & $7(6.9)$ & \\
\hline Pituitary adenoma and/or apoplexy & $29(8.8)$ & $14(13.9)$ & \\
\hline Chiari malformation & $11(3.3)$ & $2(2.0)$ & \\
\hline Other\& & $30(9.1)$ & $11(10.9)$ & \\
\hline Follow-up (incl. post-operative) & $217(66.0)$ & $53(52.5)$ & \\
\hline Meningioma & $68(20.7)$ & $20(19.8)$ & \\
\hline Vestibular schwannoma & $56(17.0)$ & $10(9.9)$ & \\
\hline Pituitary adenoma and/or apoplexy & $51(15.5)$ & $17(16.8)$ & \\
\hline Chiari malformation & $3(0.9)$ & $0(0.0)$ & \\
\hline Other \& & $39(11.9)$ & $6(5.9)$ & \\
\hline \multicolumn{4}{|l|}{ Treatment recommendation } \\
\hline Meningioma, total & 93 & 34 & $p<0.05$ \\
\hline Intervention (surgery/SRS ${ }^{\mathrm{a}}$ ), \% & $9(5 / 4)(9.7)$ & $9(7 / 2)(26.5)$ & \\
\hline Interval imaging, $\%$ & $84(90.3)$ & $25(73.5)$ & \\
\hline Vestibular schwannoma, total & 73 & 17 & $p>0.05$ \\
\hline Intervention (surgery/SRS ${ }^{\mathrm{a}}$ ), \% & $9(6 / 3)(12.3)$ & $2(1 / 1)(11.8)$ & \\
\hline Interval imaging, $\%$ & $64(87.7)$ & $15(88.2)$ & \\
\hline Pituitary adenoma/apoplexy, total & 80 & 31 & $p>0.05$ \\
\hline Surgery, $\%$ & $16(20.0)$ & $3(9.7)$ & \\
\hline Interval imaging, $\%$ & $64(80.0)$ & $28(90.3)$ & \\
\hline
\end{tabular}

*Ependymoma, nerve sheath tumour, haemangioblastoma, arachnoid/colloid cyst, etc.

${ }^{a}$ Stereotactic radiosurgery

${ }^{\&}$ Chondrosarcoma, chordoma, craniopharyngioma, etc. 
$1.1 \%$; Table 1). Within emergency operations, 30 -day perioperative mortality was lower during COVID-19 $(1.9 \%, n=3)$ compared to pre-COVID-19 $(2.5 \%, n=5)$, partly reflecting the process of patient selection with higher threshold for transfer and surgery in critically ill patients during the COVID-19 period. The single elective mortality in the COVID-19 period related to a 28 -year-old patient with a solitary CM who subsequently passed away due to leptomeningeal disease (Table 1; 30-day perioperative elective mortality during COVID-19 $n=1,2.2 \%$ ).

There were 17 neurosurgical patients who were diagnosed with COVID-19 either pre-operatively $(n=4)$ or postoperatively (operation pre-COVID-19 $n=7$, operation during COVID-19 $n=6$; all patients tested negative before surgery; Table 1), representing $2.6 \%$ of total neurosurgical operations. Out of these 17 patients, $6(35.3 \%)$ were from a black and minority ethnic (BAME) background (Table 6) and one of these BAME patients died of post-operative COVID-19 infection (accounting for $20.0 \%$ of all deaths after emergency operation). This was an 86-year-old Asian man with hypertension who underwent burr hole drainage of a chronic subdural hematoma but developed COVID-19 infection 15 days postoperatively and died 6 days later of COVID-19-related pneumonia. This was the only single mortality of a neurosurgical patient with COVID-19 infection within our cohort. There was no difference in the ethnic mix of our patients between the pre-COVID and COVID periods. The median age amongst these 17 patients was $63 \pm 15.44$ years and male:female ratio was $10: 7$. Overall, $n=4$ patients $(23.5 \%)$ were admitted to ITU because of COVID-19-related complications. The majority of patients who were infected with COVID-19 had underlying co-morbidities such as hypertension and diabetes mellitus, and all patients admitted to ITU had underlying health problems. Out of the 13 patients who developed post-operative COVID-19 infection, $53.8 \%(n=7)$ had suffered from a post-operative complication ( $n=6$ wound infection, $n=1$ hematoma, $n=1$ CSF leak) with a median time to post-operative infection of $18 \pm 9.5$ days. The median length of stay for the 17 patients diagnosed with COVID-19 was $36 \pm 23.97$ days; 4 (23.5\%) were discharged to a rehabilitation unit, and 11 (64.7\%) were discharged home.

\section{Discussion}

\section{Impact of COVID-19 on neurosurgical referrals and service}

Overall, we saw a reduction in acute referrals during COVID19 by approximately $33.6 \%$ and in the number of operations performed by approximately $55.6 \%$. This is comparable to the published literature where a reduction of more than $50 \%$ has been described by 226 respondents from more than 60 countries [9]. Mathiesen et al. demonstrated in a European snapshot that in $80 \%$ of respondents ( 20 neurosurgical departments), neurosurgical beds and neuro-intensive care beds were rationalized by postponing elective surgery, fewer acute traumatic brain injuries and subarachnoid haemorrhages admissions, and changing surgical indications in order to ration resources [14]. Although we did not see a statistically significant difference in patients with neurological deficit, GCS $\leq 8$, or age $>65$ years being transferred to our neurosurgical unit during COVID-19, there was a trend towards admitting fewer elderly patients with depressed GCS. This may represent the tendency to protect the ventilated ITU bed capacity by limiting the admission of patients with extremely poor prognosis. Additionally, referrals to our neurosurgical subspecialist MDTs were decreased although subspecialties with a more elective case mix (skull base, spine, functional) were worse affected than those with a more urgent case mix (neuro-oncology, neurovascular, paediatrics).

Hecht et al. described a similar percentage reduction for neurosurgical emergency admissions although across all subspecialties $(p=0.0007)$ during COVID-19 [6]. In keeping with their findings, we observed a reduction in the total numbers of patients presenting with sub-/epidural hematomas, traumatic vertebral fractures, and hydrocephalus (as described in Table 2). Interestingly, operations for cauda equina syndrome and spinal myelopathy decreased during COVID-19, which could be either due to a delay in presentation to the emergency department (patient-related factors) or due to a delay in referral through the general practitioner as many had their practice disrupted during COVID-19 (physician-related factors). In contrast though, we observed a similar number of patients with neurovascular emergencies (i.e. ruptured intracranial aneurysm) presenting during and pre-COVID-19 with a higher proportion undergoing endovascular treatment during COVID-19; the treatment decision was not based on resource allocation but was merely a result of the type of aneurysms presenting during the COVID-19 period. However, this also helped to reduce theatre usage and length of hospital stay.

Although there was a reduction in the referrals to our subspecialist MDTs, we developed strategies to avoid critical delays. For example, in neuro-oncology, we actively reached out to our referring centres to encourage continued referral of patients. Importantly, there was no significant change in treatment recommendation with regard to gliomas and CMs in our cohort ( $p>0.05$; Table 5). In terms of delivery of surgery, we mitigated the effects of reduced theatre space during COVID19 in our unit by securing additional theatre capacity in the private healthcare sector, contracted through the NHS. This also meant that the operations performed during COVID-19 in the private sector $(n=26)$ were provided by a "clean" neurosurgical team to a "clean" (COVID-19 negative) cohort of neuro-oncology patients, further reducing the risks. Similar 


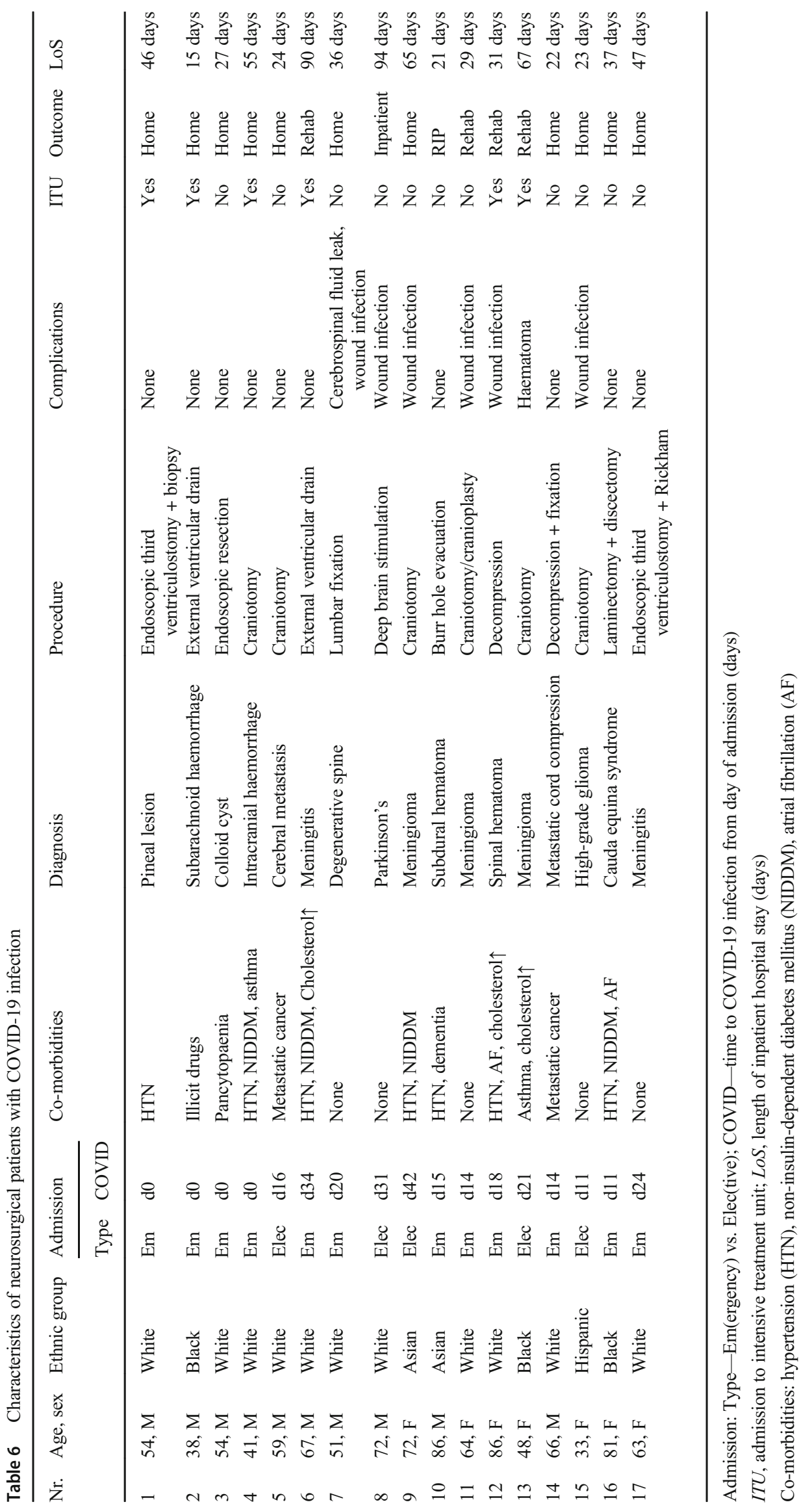


arrangements were used to maintain the delivery of the degenerative spine disease for medically refractory neural compression, with no significant difference in waiting times between pre-COVID-19 and COVID-19 periods, matching the capacity to referrals $(p>0.05$, median waiting times 28 and 27 weeks respectively).

\section{Impact of COVID-19 on subspecialties and neurosur- gical training}

There has been little focus on the impact of COVID-19 on neurosurgical training [1]. During the COVID-19, and as part of restructuring the services to release capacity to deal with COVID-19, most of our neurosurgical trainees were redeployed to ITU or COVID-19 wards. This combined with the fact that fewer operations were performed, meant a reduction in training opportunities. There was a move to teach through Zoom (Communications Technology Company, San Jose, CA) conference calls within our unit as elsewhere [19] to keep theoretical knowledge up-to-date; however, this cannot replace actual operative experience. This is further confounded as certain subspecialties have had to modify their operative techniques based on recommendations from various neurosurgical societies, particularly to avoid approaches through the respiratory tract (e.g. trans-sphenoidal surgery) or to limit the use of aerosol-generating instruments (including drills, ultrasonic aspirator) $[3,7]$.

As part of an effort to reduce physical contacts, in our unit, face-to-face outpatient clinic appointments were almost exclusively changed to telephone. The majority of telephone clinics were for patients being under regular follow-up with stable imaging findings and clinical course. All postponed or cancelled elective patients were equally kept under close telephone follow-up and prioritized for re-scheduling according to disease/symptom severity. Follow-up outcomes included further telephone consultations, repeat imaging, face-to-face assessment, or rescheduled surgery. None of these patients required emergency admission. Only patients who required neurosurgical intervention were seen in face-to-face clinic in order to be pre-assessed for surgery. Overall, this system worked well and may have potential implications on outpatient management for the future $[4,10]$. In our experience, remote access platforms such as Attend Anywhere ${ }^{\circledR}$ (Melbourne, Australia) or secure patient online chat-rooms such as The Brain Tumour Charity's BRIAN [23] provide invaluable tools in keeping contact with patients.

\section{COVID-19-positive patients and perioperative mortality}

The GlobalSurg reported a 30-day perioperative mortality of $23.8 \%$ amongst COVID-19 patients undergoing emergency or elective surgery [2]. In contrast, within our cohort, 30-day perioperative mortality remained low during COVID-19 $(2.0 \%)$. In fact, none of our four neurosurgical patients that underwent surgery whilst infected with COVID-19 died. We noted that 13 patients $(2.0 \%)$ who underwent neurosurgery before and during COVID-19 were infected with COVID-19 whilst being an inpatient; however, no single factor could be identified to trace the cause of these post-operative inpatient infections. Importantly, the majority of these patients had underlying co-morbidities or suffered a post-operative complication, hence making them more susceptible to COVID-19 infection [5]. There is new evidence suggesting that people from a BAME background are more severely affected by COVID$19[8,11,17,18] .35 .3 \%$ of our patients with COVID-19 were from a BAME background, of which one died. More data and larger cohorts are needed to further study this aspect.

\section{Limitations of this study}

Patient numbers were limited due to the relatively short time period observed. This was however inevitable and a reflection of the dynamic nature of the pandemic since the last day of recruitment was dictated by a national change in strategy towards the next phase to restore services. Our retrospective data collection for the pre-COVID-19 phase may also be another limitation here. We further did not analyse the impact of COVID-19 on excess/indirect neurosurgical mortality due to lack of presentation to hospitals such as the case maybe, for example, for ruptured aneurysms.

\section{Conclusions}

There was a reduction in neurosurgical referrals by $33.6 \%$ and operations by $55.6 \%$ during the course of COVID-19. The 30day perioperative mortality, however, remained low at $2.0 \%$, considerably lower than that in other published series [2], with the majority of patients who contracted post-operative COVID-19 infection having underlying co-morbidities and/ or suffering from post-operative complications. Despite the challenges, capacity to treat patients requiring urgent or emergency neurosurgical care was maintained at all times. The strategies we adopted allowed creation of new capacity and safe delivery of neurosurgical care, with restructuring the patient pathways and facilities into COVID-19 positive and nonCOVID-19, arguably as the most important step. We strongly believe our multi-modal approach was the key to minimize the disruptions, complications, and mortality and that lessons learned will have direct relevance for neurosurgical care during the current and future pandemics.

Contributors All authors made substantive intellectual contributions to the development of this research. KA, JJ, AMV, and AR contributed to study conception and development, data collection and data 
interpretation, and critical revision of the manuscript. All other authors contributed to study conception and development, and critical revision of the manuscript, and approved the final version of this manuscript. The corresponding author attests that all listed authors meet authorship criteria and that no others meeting the criteria have been omitted. $\mathrm{JJ}$ is the guarantor.

\section{Compliance with ethical standards}

Conflict of interest The authors declare that they have no conflict of interest.

Ethical approval All procedures performed in studies involving human participants were in accordance with the ethical standards of the institutional and/or national research committee (King's College Hospital clinical governance approval has been obtained for this audit) and with the 1964 Helsinki declaration and its later amendments or comparable ethical standards. For this type of study, formal consent is not required.

Open Access This article is licensed under a Creative Commons Attribution 4.0 International License, which permits use, sharing, adaptation, distribution and reproduction in any medium or format, as long as you give appropriate credit to the original author(s) and the source, provide a link to the Creative Commons licence, and indicate if changes were made. The images or other third party material in this article are included in the article's Creative Commons licence, unless indicated otherwise in a credit line to the material. If material is not included in the article's Creative Commons licence and your intended use is not permitted by statutory regulation or exceeds the permitted use, you will need to obtain permission directly from the copyright holder. To view a copy of this licence, visit http://creativecommons.org/licenses/by/4.0/.

\section{References}

1. Alhaj AK, Al-Saadi T, Mohammad F et al (2020) Neurosurgery residents' perspective on COVID-19: knowledge, readiness, and impact of this pandemic. World Neurosurg 139:e848-e858

2. Collaborative $\mathrm{C}$ (2020) Mortality and pulmonary complications in patients undergoing surgery with perioperative SARS-CoV-2 infection: an international cohort study. Lancet 396(10243):27-38

3. Germanò A, Raffa G, Angileri FF et al (2020) Coronavirus disease 2019 (COVID-19) and neurosurgery: literature and neurosurgical societies recommendations update. World Neurosurg 139:e812 e817

4. Ghimire P, Lavrador JP, Onyiriuka L, et al (2020). Letter: Patientreported experience measure for neuro-oncology telephone clinics during the COVID-19 pandemic. Clin Oncol (R Coll Radiol). 2020; https://doi.org/10.1016/j.clon.2020.08.004.

5. Guo G, Ye L, Pan K et al (2020) New insights of emerging SARSCoV-2: epidemiology, etiology, clinical features, clinical treatment, and prevention. Front Cell Dev Biol 8:410

6. Hecht N, Wessels L, Werft FO et al (2020) Need for ensuring care for neuro-emergencies-lessons learned from the COVID-19 pandemic. Acta Neurochir (Wien) 162(8):1795-1801

7. Iorio-Morin C, Hodaie M, Sarica C et al (2020) Letter: the risk of COVID-19 infection during neurosurgical procedures: a review of severe Acute respiratory distress syndrome coronavirus 2 (SARSCoV-2) modes of transmission and proposed neurosurgery-specific measures for mitigation. Neurosurgery 87(2):E178-E185
8. Jean WC, Ironside NT, Sack KD et al (2020) The impact of COVID-19 on neurosurgeons and the strategy for triaging nonemergent operations: a global neurosurgery study. Acta Neurochir (Wien) 162(6):1229-1240

9. Ji Y, Ma Z, Peppelenbosch MP et al (2020) Potential association between COVID-19 mortality and health-care resource availability. Lancet Glob Health 8(4):e480

10. Khalafallah AM, Jimenez AE, Lee RP et al (2020) Impact of COVID-19 on an Academic Neurosurgery Department: the Johns Hopkins Experience. World Neurosurg 139:e877-e884

11. Khunti K, Singh AK, Pareek M et al (2020) Is ethnicity linked to incidence or outcomes of covid-19? BMJ 369:m1548

12. King's College Hospital NHS Foundation Trust (2020) KCH neurosurgery COVID-19 operational guidance, V1.2, 22.04.20. In. Vol V1.2: King's College Hospital NHS Foundation Trust; 2020:1-81

13. King's College Hospital NHS Foundation Trust (2020) The King's story. https://www.kch.nhs.uk/about/the-kings-story. Accessed 12 June 2020

14. Mathiesen T, Arraez M, Asser T, et al (2020) A snapshot of European neurosurgery December 2019 vs. March 2020: just before and during the Covid-19 pandemic. Acta Neurochir (Wien) 113

15. National Health Service England (2020) Coronavirus guidance for clinicians and NHS managers. https://www.england.nhs.uk/ coronavirus. Accessed 12 June 2020

16. Office for National Statistics (2011) Census: key statistics for local authorities in England and Wales. https://www.ons.gov.uk/ peoplepopulationandcommunity/populationandmigration/ populationestimates/datasets/2011 censuskeystatisticsforlocal authoritiesinenglandandwales. Accessed 12 June 2020

17. Pan D, Sze S, Minhas JS et al (2020) The impact of ethnicity on clinical outcomes in COVID-19: A systematic review. E Clin Med 23:100404

18. Pareek M, Bangash MN, Pareek N et al (2020) Ethnicity and COVID-19: an urgent public health research priority. Lancet. 395(10234):1421-1422

19. Pennington Z, Lubelski D, Khalafallah AM et al (2020) Letter to the Editor "Changes to neurosurgery resident education since onset of the COVID-19 pandemic". World Neurosurg 139:734-740

20. Public Health England (2020) Collection COVID-19: guidance for health professionals. Information on COVID-19, including guidance on the assessment and management of suspected UK cases. https://www.gov.uk/government/collections/wuhan-novelcoronavirus. Accessed 12 June 2020

21. Sadik M (2018) Equality and equity Health profiles and demographics in Lambeth/Southwark https://www.guysandstthomas. nhs.uk/about-us/equality/objectives.aspx. Accessed 12 June 2020

22. Society for British Neurological Surgeons (2020) SBNS Covid-19 bulletins / guidance documents Society for British Neurological Surgeons. https://www.sbns.org.uk/index.php/about-us/news. Published 2020. Accessed 12 June 2020

23. The Brain Tumour Charity (2020) BRIAN. https://www. thebraintumourcharity.org/living-with-a-brain-tumour/brian. Accessed 12 June 2020

24. Vandenbroucke JP, von Elm E, Altman DG et al (2007) Strengthening the Reporting of Observational Studies in Epidemiology (STROBE): explanation and elaboration. PLoS Med 4(10):e297

Publisher's note Springer Nature remains neutral with regard to jurisdictional claims in published maps and institutional affiliations. 